Article

\title{
Multi-Objective Design Optimization of an Over-Constrained Flexure-Based Amplifier
}

\author{
Yuan Ni ${ }^{1, *}$, Zongquan Deng ${ }^{1}$, Junbao Li ${ }^{2}$, Xiang Wu ${ }^{1}$ and Long $\mathrm{Li}^{3}$ \\ 1 Research Center of Aerospace Mechanism and Control, Harbin Institute of Technology, \\ Harbin 150080, China; E-Mails: denzq@hope.hit.edu.cn (Z.D.); xiangwu@hit.edu.cn (X.W.) \\ 2 Department of Automatic Test and Control, Harbin Institute of Technology, Harbin 150001, China; \\ E-Mail: junbaolihit@gmail.com \\ 3 Shanghai Key Laboratory of Mechanical Automation and Robotic, Shanghai University, \\ Shanghai 200444, China; E-Mail: li1@shu.edu.cn \\ * Author to whom correspondence should be addressed; E-Mail: niy19831109@gmail.com; \\ Tel./Fax: +86-451-8641-3857.
}

Academic Editor: Henning Fernau

Received: 14 April 2015 / Accepted: 25 June 2015 / Published: 8 July 2015

\begin{abstract}
The optimizing design for enhancement of the micro performance of manipulator based on analytical models is investigated in this paper. By utilizing the established uncanonical linear homogeneous equations, the quasi-static analytical model of the micro-manipulator is built, and the theoretical calculation results are tested by FEA simulations. To provide a theoretical basis for a micro-manipulator being used in high-precision engineering applications, this paper investigates the modal property based on the analytical model. Based on the finite element method, with multipoint constraint equations, the model is built and the results have a good match with the simulation. The following parametric influences studied show that the influences of other objectives on one objective are complicated. Consequently, the multi-objective optimization by the derived analytical models is carried out to find out the optimal solutions of the manipulator. Besides the inner relationships among these design objectives during the optimization process are discussed.
\end{abstract}

Keywords: mechanical amplifier; multi-objective optimization; analytical model 


\section{Introduction}

In recent years, various high-precision applications have been produced and applied to prompt the micro/nano-technology to develop rapidly, such as adjustable posture institution of microwave antenna, scanning tunnel microscopy, bionic mechanism, micro-gripper, etc. [1-5]. A compliant mechanism with flexure hinges is regarded as one of the best and has been implemented in these fields due to its incomparable merits including no friction, lubrication and backlash, reduced weight, low clearance, and being easy to manufacture, integrally. For specific micro-technology applications, the micro-manipulator should have the ability to amplify the output displacement of lead zironcate titanate (PZT) in order to meet the actual requirements [6,7].

In the literature, research results have been published on every aspect of the compliant mechanism. Some works are dedicated to the design and verification of the mechanical configurations. A flexure-based compliant mechanism with three degrees of freedom (3-DOF) is presented in [8]. A parallel compliant mechanism with two axis linear motion, for ultra-precise motion, is designed in [9]. By employing flexure hinges, a micro manipulator with six degrees of freedom (6-DOF) as a fiber optics aligner is presented in [10]. The design of an XY novel flexure-based stage is proposed in [11] with high placement precision. A compliant stage with high scanning speed and a relatively large range is presented in [12]. A novel parallel-kinematic XY micro-positioning system is designed with the characteristics of a large range of motion in [13]. Another flexure-based parallel manipulator is designed with 3-DOF motion in [14].

Some researchers are focused on the quasi-static properties of the micro-manipulator. An analytical model for a classic compliant mechanism is proposed in [15]; by using this model the amplification ratio, input and output stiffness can be calculated. Another mathematical model is proposed in [16] to calculate the amplification ratio by using kinematic theory and elastic beam theory. A similar work is presented in [17]; the authors discuss several previously established models and set an improved analytical model based on Euler-Bernoulli beam theory, but the result does not match well with that of FEA. The matrix method for investigating the compliant mechanism is presented in $[18,19]$. The authors in [20] designed a micro-manipulator, with multi-lever and flexure hinges, and proposed equations to assess the properties of the mechanism. A two-step amplifier is designed in [21], and Lagrange's equation is used to calculate its performance. On the other hand, researchers are also interested in the approaches to build the stiffness analytical equations of flexure hinges. The spatial stiffness matrix is built in [22] by using the finite element method. The regular circular hinge is regarded as a beam element with three nodes in [23] for the purpose of enhancing the calculation accuracy. In [24], the flexure hinge is taken as a varied cross-section in-plane beam element with two nodes. A similar work is presented in [25]; a high-order Hermite multinomial is utilized to build shape functions of three nodes of a non-prismatic beam element. The authors in [26] presented the exact shape functions through the governing differential equations, but this method has narrow application scope.

To better acquire the satisfactory comprehensive performance of the mechanism, the studies of multi-objective optimization have been introduced to solve this problem. A hybrid technique based on differential evolution algorithms is introduced to solve multi-pass turning optimization problems in [27]. In [28], a new particle swarm-based optimization method is employed to optimize vehicle crashworthiness. A new hybrid optimization approach, based on the particle swarm optimization algorithm and 
receptor-editing properties of immune systems, seeks to solve problems in both design and manufacturing areas in [29]. In [30], an optimization algorithm called "cuckoo search algorithm" is adopted for solving manufacturing optimization problems. In [31], the paper firstly developed a mathematical model and then, combined with a multi-objective genetic algorithm, the researchers obtain the optimal design parameters of the plant. After established an analytical model, a multi-objective optimization method is employed to search for the optimal solution in [32].

The aim of this paper is to propose both of the quasi-static calculation model and the dynamic calculation model for assessing the micro-mechanism properties. Firstly, according to the theory of statically indeterminate structure, the uncanonical linear homogeneous equations are built to obtain the amplification ratio and input stiffness of the manipulator. Then, the modal analysis is carried out by the analytical dynamic model. Finally, based on these two tested theoretical models, the multi-objective optimization design is performed to improve the micro-manipulator performance.

\section{Analytical Quasi-Static Modeling}

The main component parts of the classic monolithic micro-manipulator are eight regular, circular flexure hinges. As shown in Figure 1a, the displacement loads and all of the hinges and levers are symmetric, and a 1/2 model is separated as the analytical objective, as shown in Figure $1 \mathrm{~b}$.

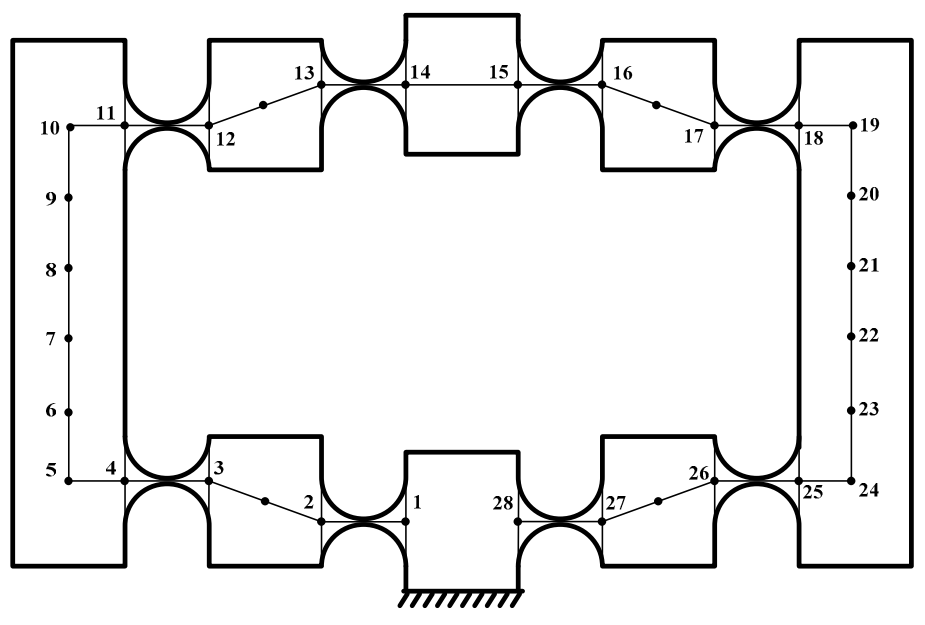

(a)

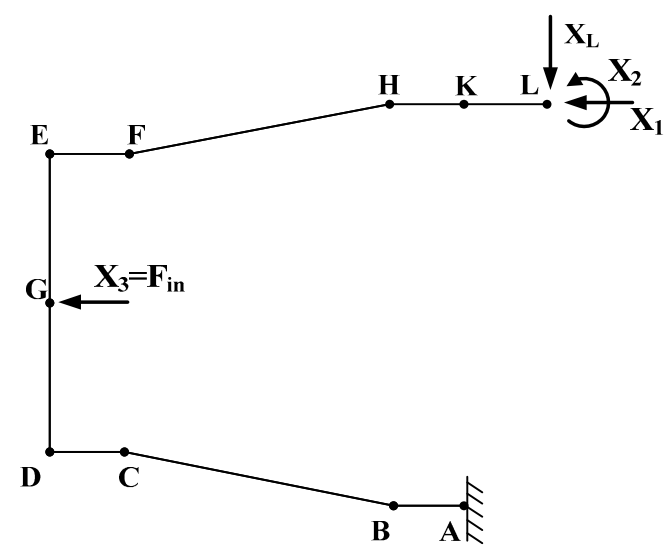

(b)

Figure 1. A displacement mechanical amplifier (a) schematic of the amplifier (b) schematic of $1 / 2$ model equivalent system.

It can be seen in Figure $1 \mathrm{~b}$ that the normal force, $\mathrm{X}_{1}$, and bending moment, $\mathrm{X}_{2}$, will be considered as the redundant forces. The input force, $\mathrm{X}_{3}$, that is produced by the PZT is not a given force and the vertical displacement of the edge point of the $1 / 2$ model would be considered. Combining above conditions and constrains, the uncanonical equations of the $1 / 2$ model are established as:

$$
\left(\begin{array}{lll}
\delta_{11} & \delta_{12} & \delta_{13} \\
\delta_{21} & \delta_{22} & \delta_{23} \\
\delta_{31} & \delta_{32} & \delta_{33} \\
\delta_{\mathrm{L} 1} & \delta_{\mathrm{L} 2} & \delta_{\mathrm{L} 3}
\end{array}\right)\left\{\begin{array}{l}
\mathrm{X}_{1} \\
\mathrm{X}_{2} \\
\mathrm{X}_{3}
\end{array}\right\}=\left(\begin{array}{c}
0 \\
0 \\
\Delta_{\text {in }} \\
\Delta_{\text {out }}
\end{array}\right)
$$


where $\Delta_{i n}$ and $\Delta_{\text {out }}$ are the varied input displacement output load and the displacement of the manipulator, respectively. $\delta_{i j}$ is defined as the corresponding displacement and can be obtained as follows:

$$
\begin{gathered}
\delta_{i j}=\int \frac{\bar{M}_{i} \bar{M}_{j}}{E I} d x=\int_{A B} \frac{\bar{M}_{i}(\theta) \bar{M}_{j}(\theta)}{E I(\theta)} d \theta+\int_{C D} \frac{\bar{M}_{i}(\theta) \bar{M}_{j}(\theta)}{E I(\theta)} d \theta+\int_{D G} \frac{\bar{M}_{i}(x) \bar{M}_{j}(x)}{E I} d x \\
+\int_{G E} \frac{\bar{M}_{i}(x) \bar{M}_{j}(x)}{E I} d x+\int_{E F} \frac{\bar{M}_{i}(\theta) \bar{M}_{j}(\theta)}{E I(\theta)} d \theta+\int_{H K} \frac{\bar{M}_{i}(\theta) \bar{M}_{j}(\theta)}{E I(\theta)} d \theta
\end{gathered}
$$

where $M_{i}$ and $M_{j}$ can be obtained by bending moment expressions. $I(\theta)$ is given by:

$$
I(\theta)=\frac{E b w^{3}(\theta)}{12}=\frac{E b[2 r+t-2 r \cos (\theta)]^{3}}{12}
$$

According to Equation (1), the displacement ratio $R$ and the input stiffness $K_{\text {in }}$ can be expressed as:

$$
\begin{gathered}
R=\frac{\Delta_{\text {out }}}{\Delta_{\mathrm{in}}}=\frac{\delta_{12}^{2} \delta_{\mathrm{L} 3}-\delta_{12} \delta_{13} \delta_{\mathrm{L} 2}-\delta_{11} \delta_{22} \delta_{\mathrm{L} 3}+\delta_{11} \delta_{23} \delta_{\mathrm{L} 2}-\delta_{12} \delta_{23} \delta_{\mathrm{L} 1}+\delta_{13} \delta_{22} \delta_{\mathrm{L} 1}}{\delta_{33} \delta_{12}^{2}-2 \delta_{12} \delta_{13} \delta_{23}+\delta_{22} \delta_{13}^{2}+\delta_{11} \delta_{23}^{2}-\delta_{11} \delta_{22} \delta_{33}} \\
K_{\text {in }}=\frac{\mathrm{X}_{3}}{\Delta_{\text {in }}}=\frac{\delta_{12}^{2}-\delta_{11} \delta_{22}}{\delta_{33} \delta_{12}^{2}-2 \delta_{12} \delta_{13} \delta_{23}+\delta_{22} \delta_{13}^{2}+\delta_{11} \delta_{23}^{2}-\delta_{11} \delta_{22} \delta_{33}}
\end{gathered}
$$

\section{Analytical Dynamic Modeling}

When the micro-manipulator is applied in the specific applications, the resonance frequency of the mechanism should be considered. On the other hand, according to the transmission principle of the classic manipulator, there are eccentric distances among some nodes. The improved analytical model is established by using the Energy principle and Hamilton principle, consequently.

\subsection{Dynamic Modeling}

The Lagrange functional is written, combining with the displacement constraints of the nodes, as follows:

$$
L^{*}=\frac{1}{2} \dot{D}_{e}^{T} M \dot{D}_{e}-\frac{1}{2} D_{e}^{T} K D_{e}+D_{e}^{T} F-\lambda^{T}\left(C D_{e}-Q\right)
$$

Where $\lambda$ is the Lagrangian multiplier, and the variational expression can be given:

$$
\delta L^{*}=-\delta D_{e}^{T} M \ddot{D}_{e}-\delta D_{e}^{T} K D_{e}+\delta D_{e}^{T} F-\delta \lambda^{T}\left(C D_{e}-Q\right)-\lambda^{T} C \delta D_{e}=0
$$

Considering the condition that:

$$
\lambda^{T} C \delta D_{e}=\delta D_{e}^{T} C^{T} \lambda
$$

and Equation (7) is rewritten as follows:

$$
\left(\begin{array}{ll}
\delta D_{e}^{T} & \delta \lambda^{T}
\end{array}\right)\left[\begin{array}{cc}
M & \mathbf{0} \\
\mathbf{0} & \mathbf{0}
\end{array}\right]\left(\begin{array}{c}
\ddot{D}_{e} \\
\ddot{\lambda}
\end{array}\right)+\left(\begin{array}{ll}
\delta D_{e}^{T} & \delta \lambda^{T}
\end{array}\right)\left[\begin{array}{cc}
K & C^{T} \\
C & \mathbf{0}
\end{array}\right]\left(\begin{array}{c}
D_{e} \\
\lambda
\end{array}\right)=\left(\begin{array}{ll}
\delta D_{e}^{T} & \delta \lambda^{T}
\end{array}\right)\left(\begin{array}{c}
F \\
Q
\end{array}\right)
$$

Eliminating the right part of Equation (9), the free vibration expression is derived as:

$$
\left[\begin{array}{cc}
M & \mathbf{0} \\
\mathbf{0} & \mathbf{0}
\end{array}\right]\left(\begin{array}{c}
\ddot{D}_{e} \\
\ddot{\lambda}
\end{array}\right)+\left[\begin{array}{cc}
K & C^{T} \\
C & \mathbf{0}
\end{array}\right]\left(\begin{array}{c}
D_{e} \\
\lambda
\end{array}\right)=\mathbf{0}
$$


where $M$ and $K$ are the total mass matrix and stiffness matrix respectively, which can be obtained by the finite element method, and the matrix $C$ is consist of multipoint constraint equations.

\subsection{The Derivation of Matrix $C$}

There are two groups of constraint equations that need to be established: let nodes $i(i=3.16)$ and $j(j=2.17)$ represent a group, and $m(m=12.27)$ and $n(n=13.26)$ stand for another group. The key to establish the constraint equations is to utilize the kinematic relationships of the nodal degrees of freedom. As shown in Figure 2a, the motion of the rigid body can be described by the node $k$, then the degrees of freedom of the nodes $i$ and $j$ can be expressed by $q_{1}, q_{2}$ and $q_{3}$. The constraint equations can be obtained as:

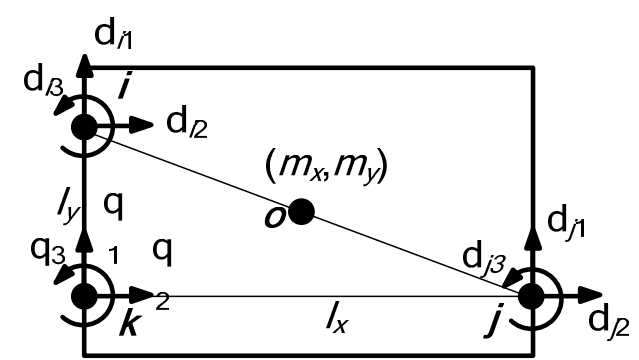

(a)

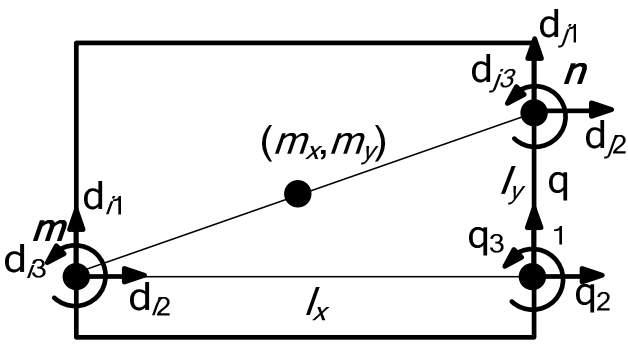

(b)

Figure 2. The rigid body with displacement offsets for multipoint constraint equations (a) $i$ and $j$ group (b) $m$ and $n$ group.

$$
\left\{\begin{array}{l}
d_{i 1}=q_{1}-l_{y} q_{3} \\
d_{i 2}=q_{2} \\
d_{i 3}=q_{3} \\
d_{j 1}=q_{1} \\
d_{j 2}=q_{2}+l_{x} q_{3} \\
d_{j 3}=q_{3}
\end{array}\right.
$$

Eliminating the degrees freedom of the rigid body $q_{1}, q_{2}$ and $q_{3}$, we can obtain three equations as:

$$
\left\{\begin{array}{l}
d_{i 1}+l_{y} d_{i 3}-d_{j 1}=0 \\
d_{i 2}+l_{x} d_{i 3}-d_{j 2}=0 \\
d_{i 3}-d_{j 3}=0
\end{array}\right.
$$

The same procedure can be performed to obtain another group of constraint equations, as shown in Figure 2b:

$$
\left\{\begin{array}{l}
d_{m 1}-l_{y} d_{m 3}-d_{n 1}=0 \\
d_{m 2}+l_{x} d_{m 3}-d_{n 2}=0 \\
d_{n 3}-d_{m 3}=0
\end{array}\right.
$$




\section{Multi-Objective Optimal Structural Design of the Mechanical Amplifier}

Parametric studies of analytical models have been carried out to investigate the influences of key design parameters on the mechanical amplifier performances, respectively [33]. On the other hand, considering this micro-manipulator may be used in some special applications, it is necessary to coordinate and compromise these major design objectives such as displacement ratio, $R$, input stiffness, $K$ in, natural frequency, $f$, the mass, $T$ and the max stress, $\sigma$, due to their complicated, variable and nonlinear relationships.

When the output displacement produced by the PZT is $0.05 \mathrm{~mm}$, the changing scopes of the cross section height, $t$, and the radius of the flexure hinge, $r$, are $0.3-0.9 \mathrm{~mm}$ and $2.2-4.5 \mathrm{~mm}$ respectively, the relationships of displacement ratio, input stiffness max stress and the first frequency are shown in Figure 3. From Figure 3a, one can find that the input stiffness, $K$ in, and the max stress, $\sigma$, is a nonlinear relationship along with the ratio, $R$, changes. For small values of the ratio, $R$, the $\sigma$ increases slowly when $K_{\text {in }}$ increases, but when $R$ changes from 0.55 to $0.65 \mathrm{~mm}$, it is noted that the $\sigma$ increases first and then decreases. In Figure $3 b$, one can see that the frequency $f$ sharp increases along with the stiffness, $K$ in, increases, but it has an inconspicuous change with the ratio, $R$. That means the objective, $f$, is more sensitive to the changes in the stiffness, $K$ in. The similar analysis processes can be carried out with another group of design parameters. When $l_{x}$ changes from 6 to $16 \mathrm{~mm}$ and $l_{y}$ changes from 2.2 to $2.9 \mathrm{~mm}$, the relationships among these design objectives are different compared with the first group. From Figure $4 \mathrm{a}$, it can be observed that the most significant change is the relationship between $\sigma$ and ratio $R$, and the frequency, $f$, is less sensitive to the changes in other objectives from Figure $4 \mathrm{~b}$. Besides, the $K_{\text {in }}$ decreases in $0.25-0.6 \mathrm{~N} / \mu \mathrm{m}$ along with $l_{x}$ and $l_{y}$ changes, this means that the ratio, $R$, and $K_{\text {in }}$ are more sensitive to the second parameter group.

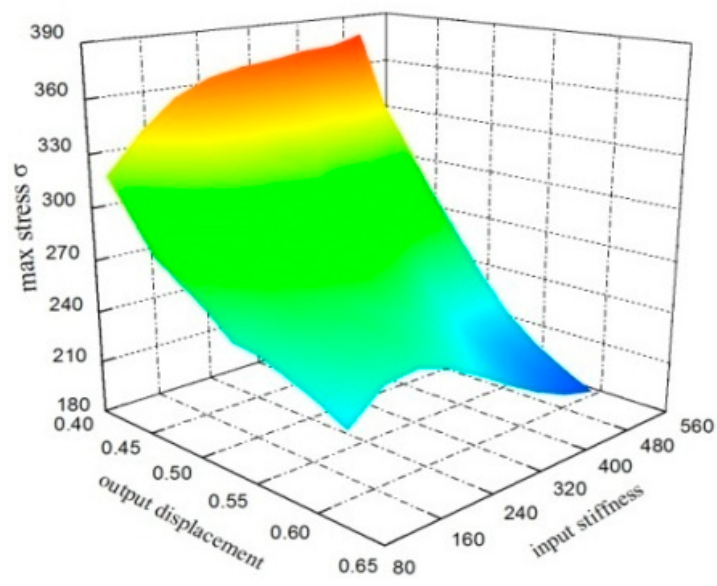

(a)

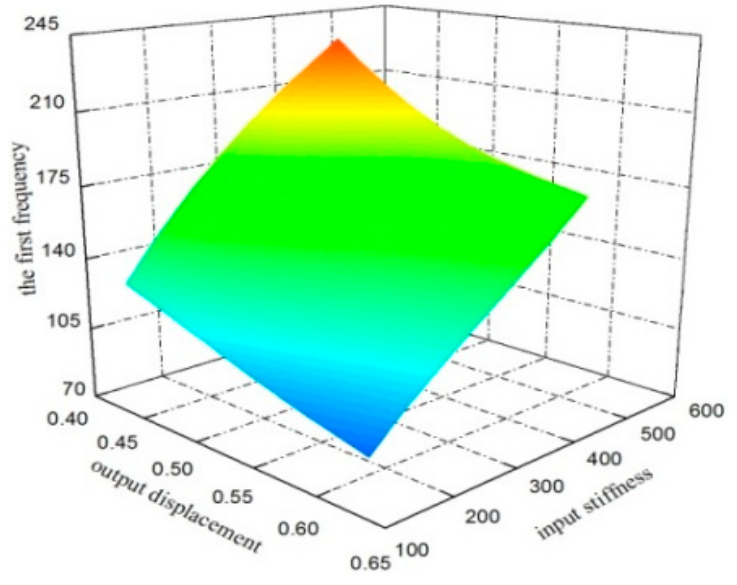

(b)

Figure 3. The relationships among the design objectives with $r$ and $t$ (a) relationships among $R-K_{\text {in }}-\sigma$ (b) relationship among $R-K_{\text {in }}-f$. 


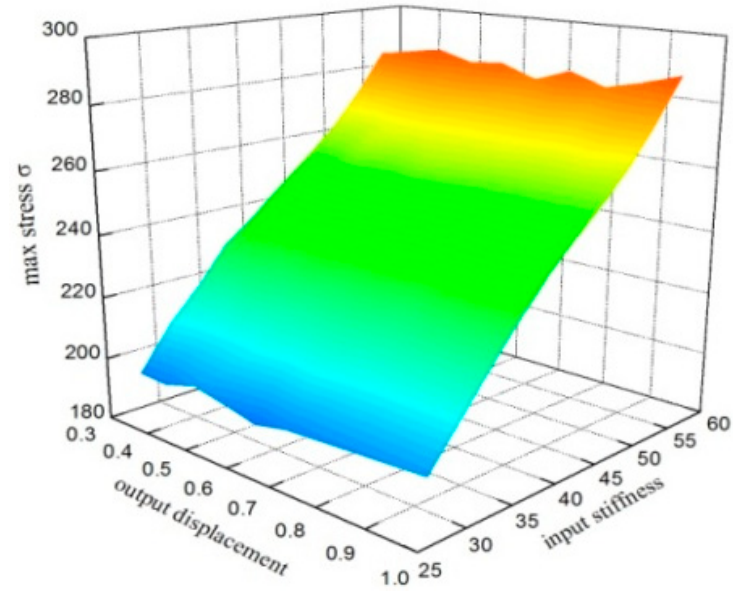

(a)

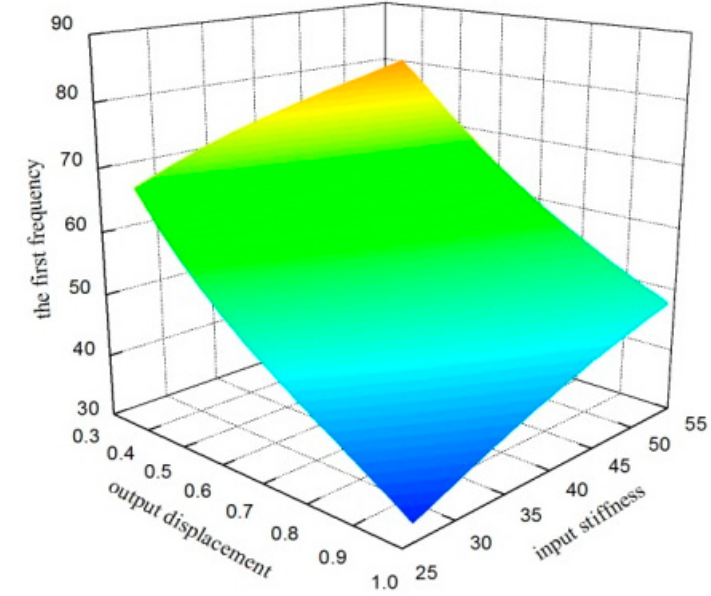

(b)

Figure 4. The relationships among the design objectives with $l_{x}$ and $l_{y}$ (a) relationships among $R-K_{\text {in }} \sigma(\mathbf{b})$ relationship among $R-K_{\text {in }}-f$.

It can be observed that the multi-objective optimization of the mechanical amplifier is complicated. In this case, the greater the displacement ratio, $R$, the better. For a given PZT, the output displacement is $0.01 \mathrm{~mm}$ and its stiffness, $K_{\mathrm{PZT}}$, has been determined; therefore the input stiffness, $K_{\text {in }}$ needs to be smaller than K КZт. One also desires that the fundamental frequency, $f$, is greater and the mass, $T$, is smaller. Consequently this model can be described as:

Max: $R, K_{\text {in }}, f$

Min: $T=4 \rho B\left\{\left(\frac{1}{2} l_{E D}+l_{f i x}\right) B_{1}+\left(l_{x}+l_{K L}\right)\left(l_{y}+2 r+t\right)+2\left[l_{x}(2 r+t)-\pi r^{2}\right]\right\}$

\section{Subject to:}

$$
\text { s.t }\left\{\begin{array}{l}
15 \mathrm{~mm} \leq l_{x} \leq 20 \mathrm{~mm} \\
2 \mathrm{~mm} \leq l_{y} \leq 3.2 \mathrm{~mm} \\
2.0 \mathrm{~mm} \leq r \leq 4.0 \mathrm{~mm} \\
0.6 \leq t \leq 1.2 \mathrm{~mm} \\
13 \leq R \leq 17 \\
80 \mathrm{~Hz} \leq f \leq 125 \mathrm{~Hz} \\
K_{\mathrm{in}} \leq K_{\mathrm{PZT}}
\end{array}\right.
$$

There are other kinds of optimization algorithms with respect to the multi-objective optimization problem. In this paper, non-dominated sorting genetic algorithm II (NSGA-II) is employed to solve this problem. The population size is 36 , the number of generations is 30 , crossover probability is 0.9 , crossover distribution is 10 , and mutation distribution is 20 , the optimized dimensions are: $l_{\mathrm{ED}}=25.51 \mathrm{~mm}, l_{x}=15.71 \mathrm{~mm}, l_{y}=2.58 \mathrm{~mm}, r=3.92 \mathrm{~mm}$, and $t=0.96 \mathrm{~mm}$, which result in this micro-manipulator with $R=14.85, f=110.72 \mathrm{~Hz}, K_{\text {in }}=11.61 \mathrm{~N} / \mu \mathrm{m}$, and $T=1.19 \mathrm{E}-4 \mathrm{~kg}$ as previously predicted by the analytical models. Figure 5 shows the game relationships among different optimal objectives. 


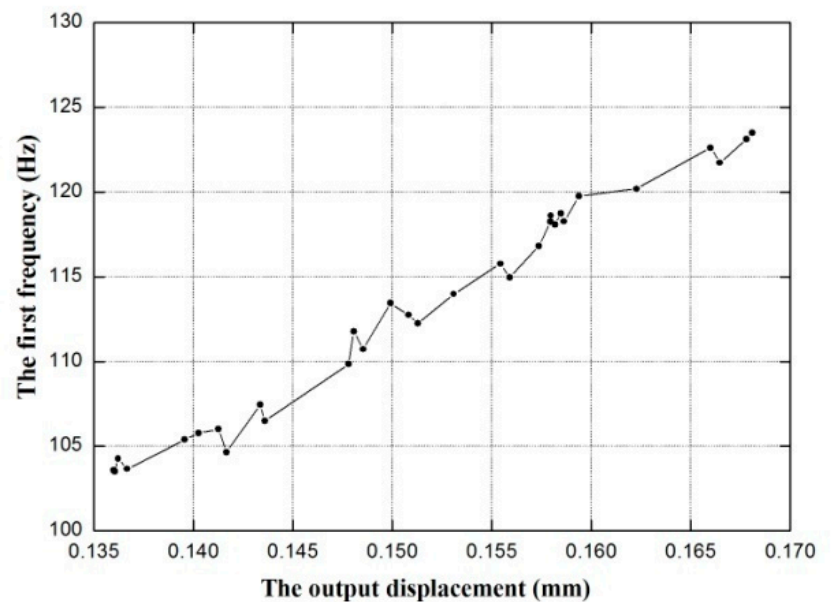

(a)

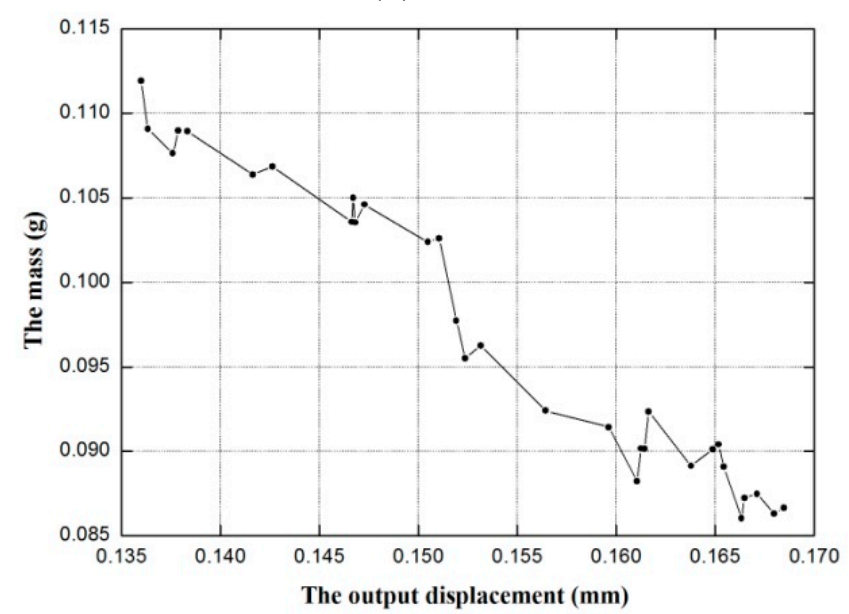

(c)

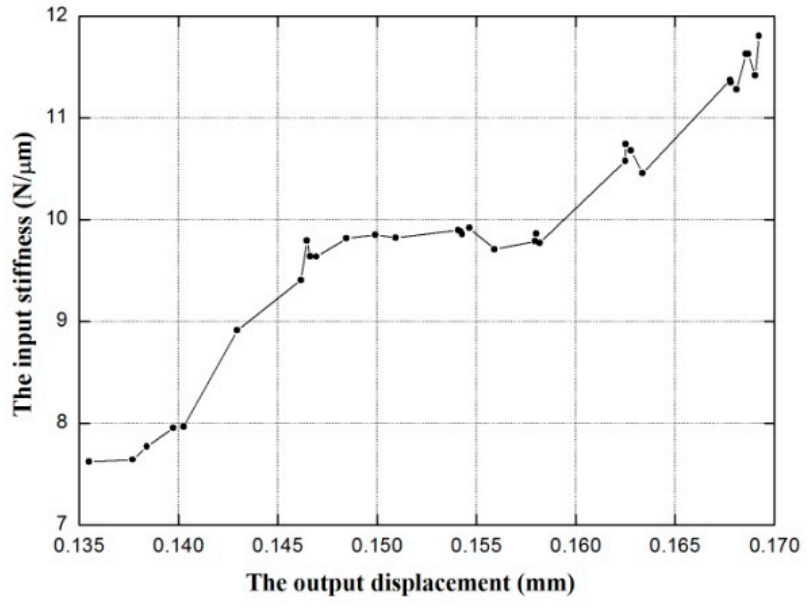

(b)

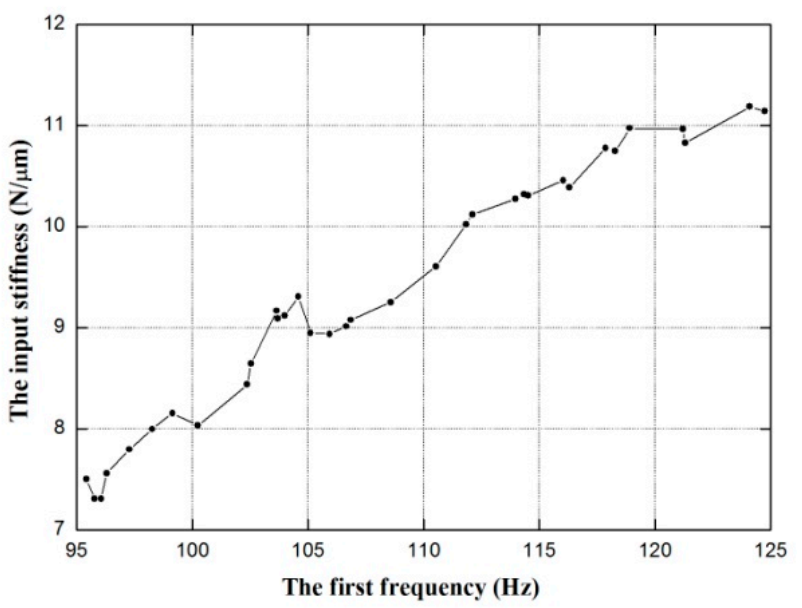

(d)

Figure 5. The game relationship among different design objectives (a) the ratio, $R$, with the frequency, $f$, (b) the ratio, $R$, with the input stiffness, $K_{\text {in, }}(\mathbf{c})$ the ratio, $R$, with the mass, $T$, (d) the frequency, $f$, with the input stiffness, $K_{\text {in. }}$.

\section{Conclusions}

This research focuses on the optimizing design of a flexure-based mechanical compliant amplifier. Based on the established and tested quasi-static analytical model and modal analytical model, the relationships among the design objectives are, consequently, discussed. It shows that the influences of other objectives on one objective are complicated. Aiming to improve the mechanical amplifier performance according to some specific applications, the multi-objective optimization of the mechanical amplifier is carried out by the NSGA-II algorithm and analytical models employed in this paper. After that, mechanical amplifier acquires satisfactory comprehensive performance.

\section{Author Contributions}

Yuan Ni organized and supervised the main research of the study. Zongquan Deng and Xiang Wu were also responsible for conceiving and designing the simulation analysis. Junbao $\mathrm{Li}$ and Long $\mathrm{Li}$ contributed the details of the algorithms. 


\section{Conflicts of Interest}

The authors declare no conflict of interest.

\section{References}

1. Henein, S.; Aymon, C.; Bottinelli, S.; Clavel, R. Articulated structures with flexible joints dedicated to high precision robotics. In Proceedings of the International Advanced Robotics Programme: Workshop on Microrobots, Micromachines and Systems, Moscow, Russia, 24-25 November 1999; pp. 135-140.

2. Kim, D.; Kang, D.; Shim, J.; Song, I.; Gweon, D. Optimal design of a flexure hinge-based XYZ atomic force microscopy scanner for minimizing Abbe errors. Rev. Sci. Instrum. 2005, 76, doi:10.1063/1.1978827.

3. Hopkins, J.B.; Culpeper, M.L. Synthesis of multi-degree of freedom, parallel flexure system via freedom and constraint topology (FACT) - Part II: Practice. Precis. Eng. 2010, 34, 271-278.

4. $\mathrm{Xu}, \mathrm{Q}$. Design and development of flexure-based dual-stage nanopositioning system with minimum interference behavior. IEEE Trans. Autom. Sci. Eng. 2012, 9, 554-563.

5. Liaw, H.C.; Shirinaadeh, B.; Simith, J. Robust motion tracking control of piezo-driven flexure-based four-bar mechanism for micro/nano manipulation. Mechatronics 2008, 18, 111-120.

6. Xu, W.; King, T. Flexure hinges for piezoactuator displacement amplifiers: Flexibility, accuracy, and stress considerations. Precis. Eng. 1996, 19, 4-10.

7. Jouaneh, M.; Yang, R. Modeling of flexure-hinge type lever mechanisms. Precis. Eng. 2003, 27, 407-418.

8. Bhagat, U.; Shirinzadeh, B.; Clark, L. Design and analysis of a novel flexure-based 3-DOF mechanism. Mech. Mach. Theory 2014, 74, 173-187.

9. Choi, K.; Kim, D. Monolithic parallel linear compliant mechanism for two axes ultraprecision linear motion. Rev. Sci. Instrum. 2006, 77, doi:10.1063/1.2207368.

10. Chao, D.; Zong, G.; Liu, R. Design of a 6-DOF compliant manipulator based on serial-parallel architecture. In Proceedings of the 2005 IEEE/ASME International Conference on Advanced Intelligent Mechatronics, Monterey, CA, USA, 24-28 July 2005; pp. 765-770.

11. Choi, Y.J.; Sreenivasan, S.V.; Choi, B.J. Kinematic design of large displacement precision XY positioning stage by using cross strip flexure joints and over-constrained mechanism. Mech. Mach. Theory 2008, 43, 724-737.

12. Yuen, Y.; Sumeet, S. Design, Identification, and Control of a Flexure-Based XY Stage for Fast Nanoscale Positioning. IEEE Trans. Nanotechnol. 2009, 8, 46-54.

13. $\mathrm{Xu}, \mathrm{Q}$. New Flexure Parallel-Kinematic Micropositioning System with Large Workspace. IEEE Trans. Robot. 2012, 28, 478-491.

14. Teo, T.J.; Yang, G.L.; Chen, I.M. A large deflection and high payload flexure-based parallel manipulator for UV nanoimprint lithography: Part I. Modeling and analyses. Precis. Eng. 2014, $38,861-871$. 
15. Lobontiu, N.; Garcia, E. Analytical model of displacement amplification and stiffness optimization for a class of flexure-based compliant mechanism. Comput. Struct. 2003, 81, 2797-2810.

16. Ma, H.W.; Yao, S.M.; Wang, L.Q.; Zhong, Z. Analysis of the displacement amplification ratio of bridge-type flexure hinge. Sens. Actuators A Phys. 2006, 132, 730-736.

17. $\mathrm{Xu}, \mathrm{Q}$;; Li, Y. Analytical modeling, optimization and testing of a compound bridge-type compliant displacement amplifier. Mech. Mach. Theory 2011, 46, 183-200.

18. Koseki, Y.; Tanikawa, T.; Koyachi, N.; Arai, T. Kinematic analysis of translational 3-DOF micro parallel mechanism using matrix method. In Proceedings of the 2000 IEEE/RSJ International Conference on Intelligent Robots and Systems, Takamatsu, Japan, 31 October-5 November 2000; pp. 786-792.

19. Ye, G.; Li, W.; Wang, Y.; Yang, X.; Yu, L. Kinematics analysis of Bridge-type micro-displacement mechanism based on Flexure hinge. In Proceedings of the 2010 IEEE International Conference on Information and Automation, Harbin, China, 20-23 June 2010; pp. 66-70.

20. Yang, R.; Jouaneh, M.; Schweizer, R. Design and character of a low-profile micropositioning stage. Precis. Eng. 1996, 18, 20-29.

21. Choi, S.B.; Han, S.S.; Han, Y.M.; Thompson, B.S. A magnification device for precision mechanisms featuring piezoactuators and flexure hinges: Design and experimental validation. Mech. Mach. Theory 2007, 42, 1184-1198.

22. Zhang, S.; Fasse, E.D. A Finite-Element-Based Method to Determine the Spatial Stiffness Properties of a Notch Hinge. J. Mech. Des. 2001, 123, 141-147.

23. Lobontiu, N.; Garcia, E. Circular-Hinge Line Element for Finite Element Analysis of Compliant Mechanisms. J. Mech. Des. 2005, 127, 766-773.

24. Wang, H.; Zhang, X. Input coupling analysis and optimal design of a3-DOF compliant micro-positioning stage. Mech. Mach. Theory 2008, 43, 400-410.

25. Zhang, X.; Hou, W. Dynamic analysis of the precision compliant mechanisms considering thermal effect. Precis. Eng. 2010, 34, 592-606.

26. Filippo, R.; Gaetano, Z. Deflections of beams with varying rectangular cross section. J. Eng. Mech. 1992, 118, 2128-2134.

27. Yildiz, A.R. A new hybrid particle swarm optimization approach for structural design optimization in the automotive industry. J. Automob. Eng. 2012, 226, 1340-1351.

28. Yildiz, A.R.; Solanki, K.N. Multi-objective optimization of vehicle crashworthiness using a new particle swarm based approach. Int. J. Adv. Manuf. Technol. 2012, 59, 367-376.

29. Yildiz, A.R. A novel particle swarm optimization approach for product design and manufacturing. Int. J. Adv. Manuf. Technol. 2009, 40, 617-628.

30. Yildiz, A.R. Cuckoo search algorithm for the selection of optimal machining parameters in milling operations. Int. J. Adv. Manuf. Technol. 2013, 64, 55-61. 
31. Shirazi, A.; Najafi, B.; Aminyavari, M.; Rinaldi, F.; Taylor, R.A. Thermal-Economic-Environmental Analysis and Multi-objective Optimization of an Ice Thermal Energy Storage System for Gas Turbine Cycle Inlet Air Cooling. Energy 2014, 69, 212-216.

32. Mamaghani, A.H.; Najafi, B.; Shirazi, A. 4E analysis and multi-objective optimization of an integrated MCFC (molten carbonate fuel cell) and ORC (organic Rankine cycle) system. Energy 2015, 82, 650-663.

33. Ni, Y.; Deng, Z.; Wu, X. Modeling and analysis of an over-constrained flexure-based compliant mechanism. Measurement 2014, 50, 270-278.

(C) 2015 by the authors; licensee MDPI, Basel, Switzerland. This article is an open access article distributed under the terms and conditions of the Creative Commons Attribution license (http://creativecommons.org/licenses/by/4.0/). 